\title{
Relation between cholecystokinin and antral innervation in the control of gastric emptying in the rat
}

\author{
A Higham, C Vaillant, B Yegen, D G Thompson, G J Dockray
}

\begin{abstract}
Background-Antral motility and the hormone cholecystokinin (CCK) are major determinants of the rate of gastric emptying. The relation between CCK and antral neurons in regulating gastric emptying is uncertain. Benzalkonium chloride (BAC) causes selective lesions in gut myenteric neurons after serosal application

Aim-To develop a model of antral denervation using BAC to enable the study of the relation between CCK and antral neurons in regulating gastric emptying.

Methods-BAC, vehicle or the afferent neurotoxin capsaicin were applied to the serosal surface of the rat antrum or corpus; neurochemical markers of intrinsic and afferent neurons were detected by using immunohistochemistry and radioimmunoassay. Gastric retention of solids was determined after fasting, and emptying of liquids was measured in rats with gastric fistulae.
\end{abstract}

Results-In BAC treated rats radioimmunoassay of tissue extracts revealed a dose related specific loss of gastrin releasing peptide, substance $P$, and vasoactive intestinal polypeptide immunoreactivities from the treated region, and immunohistochemistry revealed loss of the neuronal marker PGP 9.5 and the afferent neuropeptide calcitonin gene related peptide (CGRP). Adjacent untreated regions were unaffected by $B A C$, with the exception that CGRP was depleted in both corpus and antrum after antral treatment. After antral BAC treatment fasted rats retained solids for over 48 hours. Moreover, in antrally denervated rats with gastric fistulae, the emptying of saline, acid and peptone was delayed substantially. The CCK dependent inhibition of gastric emptying of peptone was preserved after antral treatment with BAC.

Conclusions-Serosal BAC causes lesions in the innervation of the treated region of the stomach. The innervation of the antrum is essential for normal emptying of both liquids and solids, but the inhibition of gastric emptying produced by CCK is not dependent on antral neurons. (Gut 1997; 41: 24-32)

Keywords: antral neurons; benzalkonium chloride; cholecystokinin; gastric emptying
Gastric emptying of liquids is dependent on the pressure gradient between the lumen of the stomach and proximal duodenum, and the resistance to flow between the two. ${ }^{1}$ Emptying of liquids is pulsatile in nature and this can be attributed to phasic contraction of the pyloric antral region of the stomach, a motor pattern dependent on the antral innervation. ${ }^{2-4}$ It has long been known that the rate of emptying depends on the nature of the luminal contents; thus, acid, hyperosmolal solutions, high viscosity, protein and fat rich meals all delay gastric emptying. ${ }^{1}$ Although a substantial body of evidence has emerged to suggest that the intestinal hormone cholecystokinin (CCK) is a physiological mediator of the effect of fat and protein rich meals in delaying gastric emptying, ${ }^{5}$ the relation between CCK and antral neurons that might be relevant in controlling this process is incompletely understood.

Studies of the action of CCK in delaying gastric emptying have focused on the role of vago-vagal reflexes. In particular there is good evidence that these reflexes are initiated by the stimulation of vagal afferent neurons by CCK. ${ }^{6-8}$ Studies in this area have made extensive use of the sensory neurotoxin capsaicin. When high doses of capsaicin are administered to neonatal rats, or high concentrations applied directly to afferent nerve trunks, there is a permanent loss of small diameter afferent neurons. In both cases the CCK mediated control of gastric emptying is attenuated considerably. ${ }^{6}$ There is some evidence that CCK acts reflexly to relax the body of the stomach and thereby to decrease the pressure gradient between stomach and duodenum. ${ }^{6}{ }^{10}$ Inhibitory myenteric neurons, such as those acting via release of vasoactive intestinal polypeptide (VIP) and nitric oxide, may be relevant here. ${ }^{711} 12$ There is also evidence that CCK acts on the distal stomach, both by direct stimulation of smooth muscle and indirectly via release of neurotransmitters. ${ }^{13}$ However, the importance of the latter effect for the control of gastric emptying is unknown.

This study was undertaken to determine the role of the antral innervation in the control of gastric emptying. The initial aim of the study was to develop an experimental model in which lesions can be caused selectively in the antral innervation. To achieve this, the detergent benzalkonium chloride (BAC) was used, which has already been shown to cause lesions in small intestinal myenteric neurons. ${ }^{14} 15$ 
Complete loss of the innervation of the antrum caused notable gastric retention of solids. To define the effects of antral denervation on mechanisms regulating the gastric emptying of liquids more clearly, rats treated with BAC were fitted with a gastric cannula. This permitted the study of gastric emptying of a variety of liquid test solutions under different experimental conditions.

\section{Methods}

ANIMALS

Adult Wistar rats (approximately $250 \mathrm{~g}$ ) were kept on a 12 hour light/dark cycle. Rats were fasted overnight before operations but allowed free access to water. They were anaesthetised by intramuscular injection of a mixture of fentanyl citrate $(0.32 \mathrm{mg} / \mathrm{kg})$, fluanisone $(4 \mathrm{mg} /$ $\mathrm{kg})$ and diazepam $(2 \mathrm{mg} / \mathrm{kg})$.

\section{TREATMENT WITH BAC}

The stomach was exteriorised through a midline upper abdominal incision. The serosal surface of the region to be denervated was covered with a layer of gauze cut to size. Benzalkonium chloride $(0.02 \%, 0.1 \%$, or $0.5 \%$ $\mathrm{w} / \mathrm{v}$ ) dissolved in $0.9 \% \mathrm{NaCl}$ was painted onto the gauze, which was kept moist with the BAC solution for three minutes, and then removed. The serosal surface of the stomach was then rinsed thoroughly with normal saline and returned to the peritoneal cavity, and the incision closed in layers. The two faces of the stomach were treated separately; in some experiments only the anterior face of the antrum was treated. A similar method was used for denervation of the corpus using a strip of gauze (approximately $5 \mathrm{~mm}$ wide) placed over the corpus alongside the border of the glandular and non-glandular regions. In order to cause selective lesions of the afferent innervation of the stomach, the same experimental approach was used, using the sensory neurotoxin capsaicin $(1 \% \mathrm{w} / \mathrm{v}$ in olive oil) in place of BAC. Control animals underwent a sham operation with serosal application of normal saline (or olive oil). Animals were allowed to recover for at least 11 days before experiments.

EXPERIMENTAL STUDIES

Initial studies were undertaken to define the optimal dose of BAC, and the time course of subsequent responses. Denervation was assessed by radioimmunoassay of neuropeptides in extracts of BAC treated and adjacent tissues, and by immunohistochemistry. Gastric emptying was meaured in two separate studies. Firstly, the dry weight of solids retained in the stomach was measured in rats fed standard chow ad libitum and then fasted for 48 hours on wire bottomed cages. Rats were killed by cervical dislocation followed by decapitation, trunk blood was taken for assay of fasting plasma CCK, and the stomach contents were removed, weighed wet and again after prolonged drying at $50^{\circ} \mathrm{C}$. The stomach was freed of adhesions, and tissue extracted for determination of neuropeptide concentration by radioimmunoassay or fixed for immunohistochemistry. Secondly, in rats with a gastric cannula, the emptying of liquids instilled into the stomach was measured as described later.

RADIOIMMUNOASSAY

The neuropeptide content of tissues was measured after extraction with boiling water $(0 \cdot 1 \mathrm{~g} / \mathrm{ml})$, homogenising and centrifuging, and re-extraction of the pellet with $0.5 \mathrm{M}$ acetic acid, as described previously. ${ }^{16}$ Extracts were assayed at the appropriate dilution for the following peptides: gastrin releasing peptide (GRP) was assayed using antibody 1078 , with porcine GRP 14-28 as standard, and iodinated $\mathrm{Tyr}^{1}$ COOH-terminal decapeptide of bombesin (Peninsula Laboratories, St Helens, $\mathrm{UK}$ ) as label ${ }^{17}$; calcitonin gene related peptide (CGRP) was assayed with antibody L273, using rat $\operatorname{Tyr}^{0} \alpha-C G R P 28-37$ as standard and

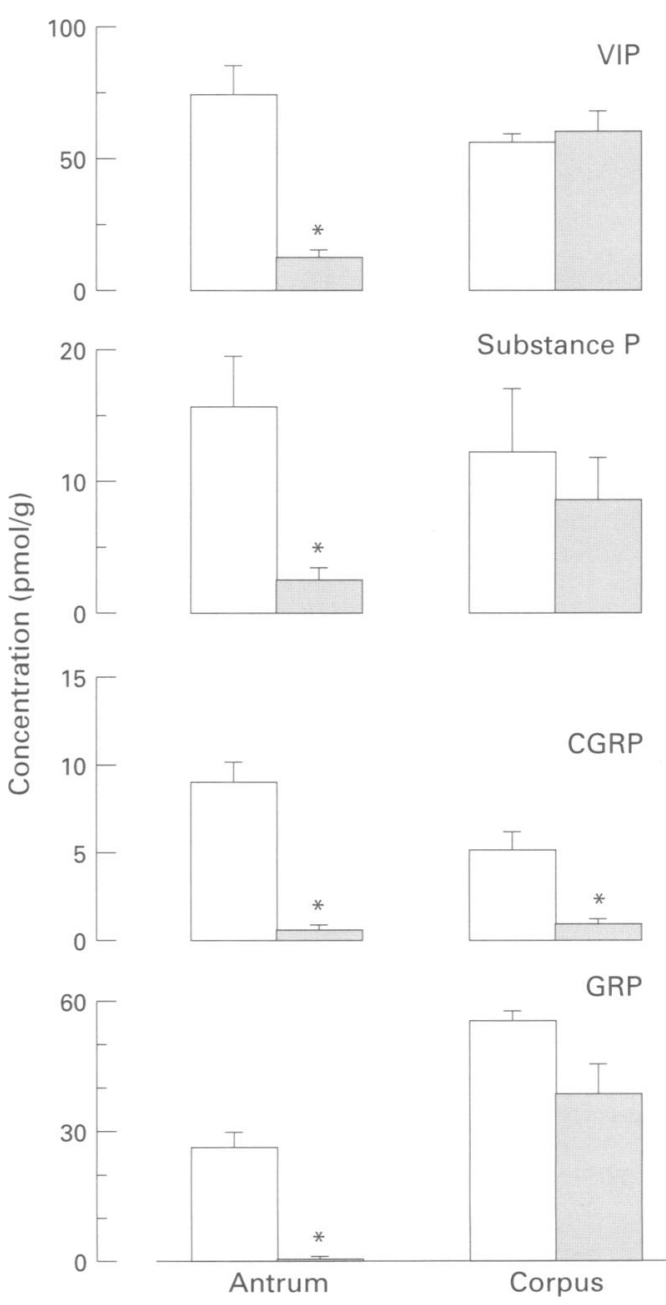

Figure 1: Neuropeptide concentrations (GRP, CGRP, substance $P$, and VIP) in extracts of antrum and corpus, after serosal application of $0.5 \%$ w/v BAC (tinted bars) or vehicle (open bars) to the antrum. After application of $B A C$ there was a significant loss of neuropeptides derived from both intrinsic and extrinsic afferent neurons of the antrum. CGRP was also significantly reduced in the corpus ( $n=6$ all groups; ${ }^{\star} p<0 \cdot 05$, compared with control, MannWhitney $U$ test). Values expressed as means (SEM). 
the same peptide labelled with ${ }^{125} \mathrm{I}$ as label ${ }^{18}$; substance $\mathrm{P}$ was assayed with antibody L83, using undecapeptide substance $P$ as standard and ${ }^{125} \mathrm{I}$-Bolton Hunter labelled substance $\mathrm{P}$ (Amersham, UK) as label; VIP was assayed using antibody L25, with porcine VIP as standard (Peninsula Laboratories), and ${ }^{125} \mathrm{I}$ labelled VIP (Amersham, UK) as label. ${ }^{16}$ Plasma CCK was assayed using antibody DINO with synthetic sulphated CCK8 as standard and Bolton-Hunter ${ }^{125} \mathrm{I}$ labelled CCK8 as label (Amersham, UK). ${ }^{19}$

IMMUNOHISTOCHEMISTRY

A strip of stomach wall from the non-glandular corpus to the pyloric sphincter was removed from animals treated by circumferential application of $0.5 \% \mathrm{w} / \mathrm{v} \mathrm{BAC}$, or saline, to the serosal surface of the antrum, or the junction of the glandular and non-glandular regions of the corpus, as described earlier. Samples were
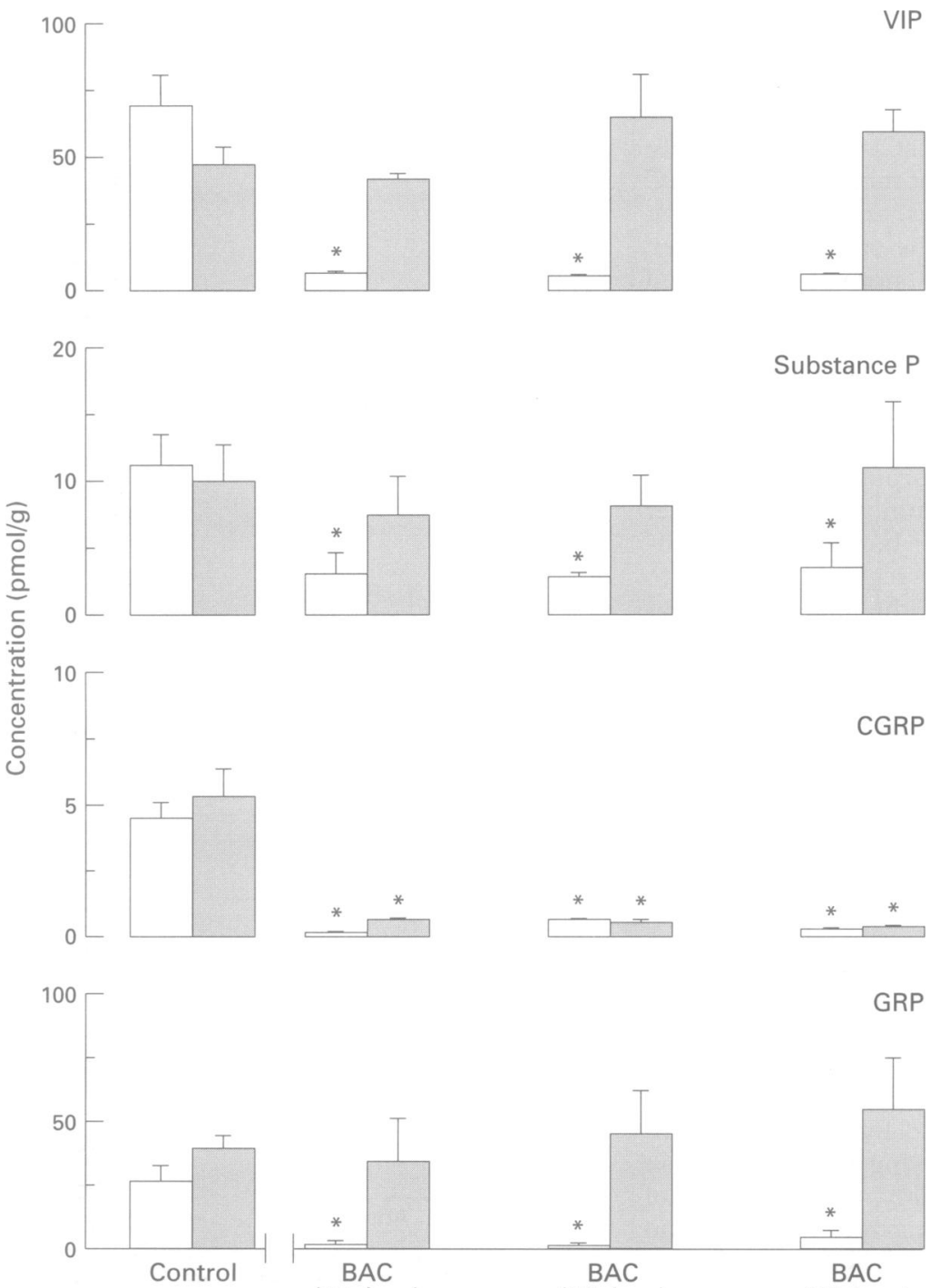

concentrations (GRP, CGRP, substance $P$, and VIP) in extracts of the antrum (open bars) and corpus (tinted bars) $11(n=5), 18(n=5)$, and $25(n=3)$ days after application of $0.5 \% \mathrm{w} / v B A C$ or vehicle (control, $n=6$ ) to the serosa of the antrum. The loss of neuropeptides was complete at day 11 and unchanged thereafter. Note again, selective loss of corpus CGRP immunoreactivity following antral denervation ${ }^{*} p<0.05$, compared with control, Mann-Whitney $U$ test). Values expressed as mean (SEM). fixed by immersion for 24 hours in $4 \%$ paraformaldehyde in $0 \cdot 1 \mathrm{M}$ sodium cacodylate buffer, and processed by cryostat sectioning. Immunoperoxidase staining for the neuronal marker protein gene product (PGP) $9 \cdot 5,{ }^{20}$ and the sensory neuropeptide CGRP was carried out by incubating sections for 18 hours at $4^{\circ} \mathrm{C}$ in rabbit anti-PGP 9.5 (UltraClone, UK) or rabbit anti-CGRP (L273), both diluted 1 in 1500. After washing, sections were incubated for one hour in biotinylated goat anti-rabbit IgG (Sigma) then in streptavidin biotinylated peroxidase complexes (Amersham, UK), both diluted 1 in 50. Sites of antibody binding were visualised with glucose-nickel-diaminobenzidine substrate. ${ }^{21}$

RATS WITH GASTRIC FISTULAE

Animals were treated by application of either BAC or saline to the antral serosa as described earlier, and a small stainless steel Gregory cannula was installed in the corpus as described previously. ${ }^{22}$ The cannula was exteriorised through a midline stab incision and the incision closed in layers. For gastric emptying studies, rats with gastric fistulae were allowed at least 11 days to recover from surgery before experiments. Prior to experiments the stomach was washed extensively with warm $0.14 \mathrm{M} \mathrm{NaCl}$ to remove solids. On the experimental day, the emptying of test solutions (saline, $50 \mathrm{mM} \mathrm{HCl}, 4 \cdot 5 \%$ w/v meat peptone II, containing phenol red, $60 \mathrm{mg} / \mathrm{l}$ ) instilled into the stomach via the gastric fistula, was determined as described previously. ${ }^{9}$ The CCK antagonist L-364,718 $(1.0 \mathrm{mg} / \mathrm{kg})$ was administered by instillation into the gastric fistula as described previously. ${ }^{23}$

\section{Results}

SEROSAL APPLICATION OF BAC TO ANTRUM Initial experiments were directed at determining the optimal dose of BAC for antral denervation. The tissue concentrations of GRP, CGRP, substance P, and VIP measured by radioimmunoassay of antral extracts were reduced in a dose dependent manner 11 days after prior administration of BAC. At a concentration of $0.02 \%$, BAC had no effect; $0 \cdot 1 \%$ BAC produced a $25 \%$ loss of CGRP, but after application of $0.5 \% \quad \mathrm{w} / \mathrm{v}$ BAC all neuropeptides were depleted (fig 1). The concentrations of GRP, substance $P$, and VIP in extracts of the gastric corpus were not affected by prior application of BAC to the antrum (fig 1). However, the concentration of CGRP in the corpus was reduced significantly by application of $0.5 \%$ BAC to the antrum.

In order to determine the duration of responses to $0.5 \%$ BAC, neuropeptide concentrations were examined in extracts of tissues sampled 11 to 25 days after treatment. All neuropeptides assayed were depleted in antral extracts 11 days after application of BAC, and remained substantially reduced at all times up to 25 days. Similarly, there was loss of CGRP 
in both antrum and corpus in rats in which the antrum had been treated with BAC at all times examined (fig 2 ).

SPECIFICITY OF BAC INDUCED DENERVATION To examine the specificity of the effects described above, we studied the effect of BAC applied to a circumferential band (approximately $5 \mathrm{~mm}$ ) of the gastric corpus immediately distal to the non-glandular part of the stomach. In the BAC treated region there was substantial depletion of all the neuropeptides studied. In the untreated corpus, and in the antrum, the tissue concentrations of GRP, CGRP, substance $P$, and VIP were similar to
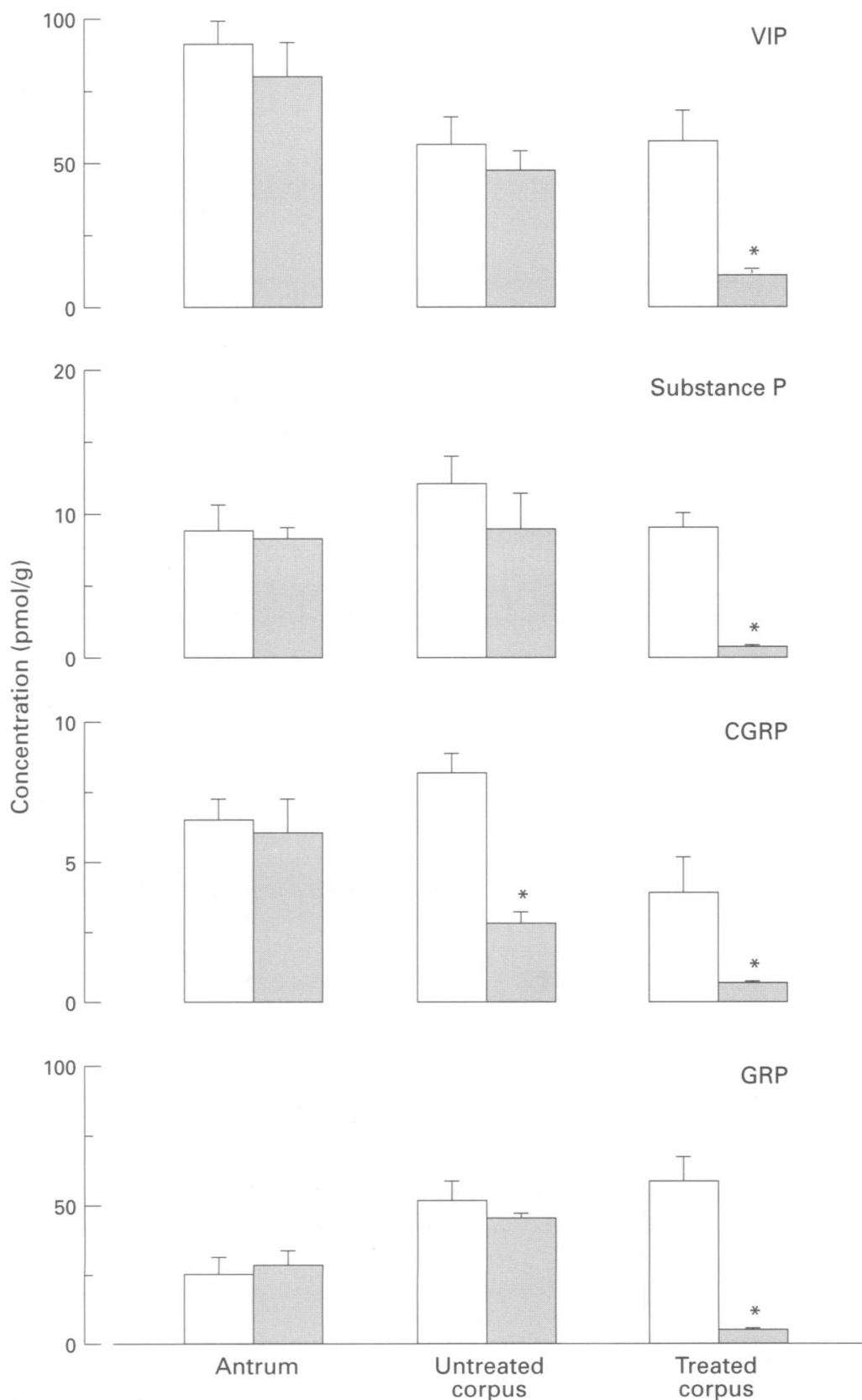

Figure 3: Neuropeptide concentrations (GRP, CGRP, substance $P$, and VIP) in extracts of antrum, control corpus and a band of proximal corpus treated with either $0.5 \%$ BAC (tinted bars) or vehicle (open bars). There was complete loss of the neuropeptides in the band of corpus exposed to BAC but not in the remainder of the corpus, or the antrum. Note in particular the preservation of CGRP in the antrum ${ }^{\star} p<0.05$, compared with vehicle, Mann-Whitney U test; $n=6$ all groups). Values expressed as mean (SEM). control values (fig 3). It is worth emphasising that following treatment of the corpus with BAC, antral CGRP concentrations were unaffected (fig 3). In addition, in some experiments BAC was applied to a single side of the antrum. In this case, there was depletion of neuropeptides on the treated side as already noted, but the concentrations in the untreated side of the antrum were similar to control values (not shown).

APPLICATION OF CAPSAICIN TO ANTRUM

The effects of the sensory neurotoxin capsaicin applied in a similar fashion to BAC, were examined in order to differentiate between loss of extrinsic afferent neurons and both intrinsic and extrinsic afferent neurons. Serosal application of $1 \% \mathrm{w} / \mathrm{v}$ capsaicin to the antrum produced complete loss of CGRP in extracts of antrum and corpus (fig 4). In contrast,

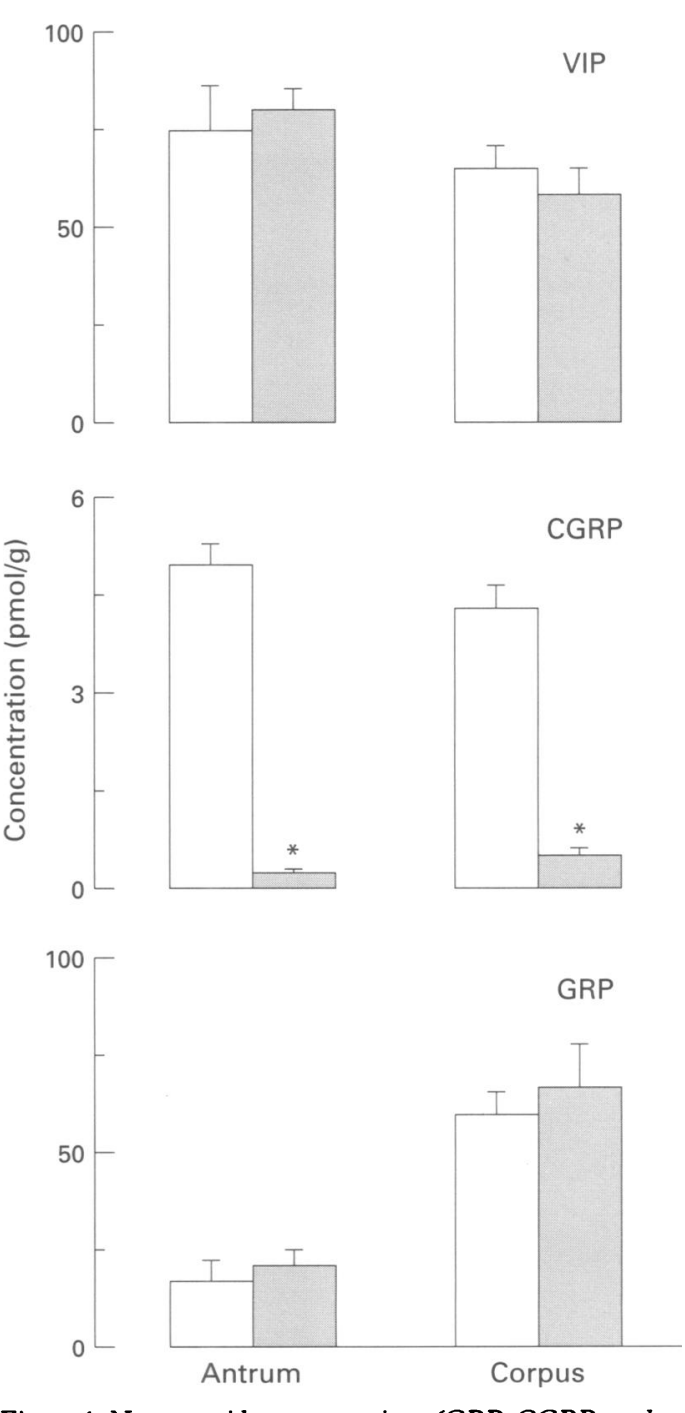

Figure 4: Neuropeptide concentrations (GRP, CGRP, and VIP) in extracts of antrum and corpus following topical application of $0 \cdot 1 \% \mathrm{w} / \mathrm{v}$ capsaicin (tinted bars) or vehicle (open bars) to the serosal surface of the antrum. The spinal afferent innervation of both the antrum and corpus was lost as shown by CGRP concentrations, whereas neuropeptides of the intrinsic innervation (GRP, VIP) were unaffected in both regions $\left(n=4\right.$, both groups; ${ }^{\star} p<0.05$, compared with both regions $\left(n=4\right.$, both groups; ${ }^{\star} p<0 \cdot 05$, compared with
vehicle, Mann-Whitney U test). Values expressed as mean (SEM). 
neuropeptides found in intrinsic neurons (GRP and VIP) were not changed by treatment of the antrum with capsaicin.

\section{IMMUNOHISTOCHEMISTRY}

The effects of BAC on the gastric innervation described above were supported by immunohistochemical studies. There was a complete loss of immunoreactive nerve cell bodies and fibres stained for the neuronal marker PGP 9.5 in the treated region only after treatment with $0.5 \%$ BAC. Both immunoreactive nerve cell bodies and nerve fibres were present in untreated regions (fig 5). Staining for CGRP showed a different pattern antral application of BAC resulted in a loss of CGRP immunoreactive fibres from both the antrum and corpus (figs $6 \mathrm{c}$ and $6 \mathrm{~d}$ ), whereas denervation of a band of corpus with BAC resulted in complete loss of immunoreactive fibres in the treated region, but not in the untreated antrum (figs $6 \mathrm{e}$ and $6 \mathrm{f}$ ).

TREATMENT OF THE ANTRUM WITH BAC CAUSES GASTRIC RETENTION OF SOLIDS

In rats treated antrally with $0.5 \%$ BAC, there was a striking retention of solids in the stomach (fig 7). The wet and dry weight of gastric contents was variable in BAC treated rats but all rats treated with $0.5 \%$ BAC exhibited overtly distended stomachs (by solid, gas or liquid) after a 48 hour fast. The retention of solids was seen 11 to 25 days after $0.5 \%$ BAC, but was not evident in rats treated with lower concentrations of BAC (results not shown). The data presented earlier indicate that BAC causes loss of both intrinsic and extrinsic neurons, whereas capsaicin causes lesions in extrinsic afferents only; we therefore used the latter to determine whether the effects of BAC might simply reflect
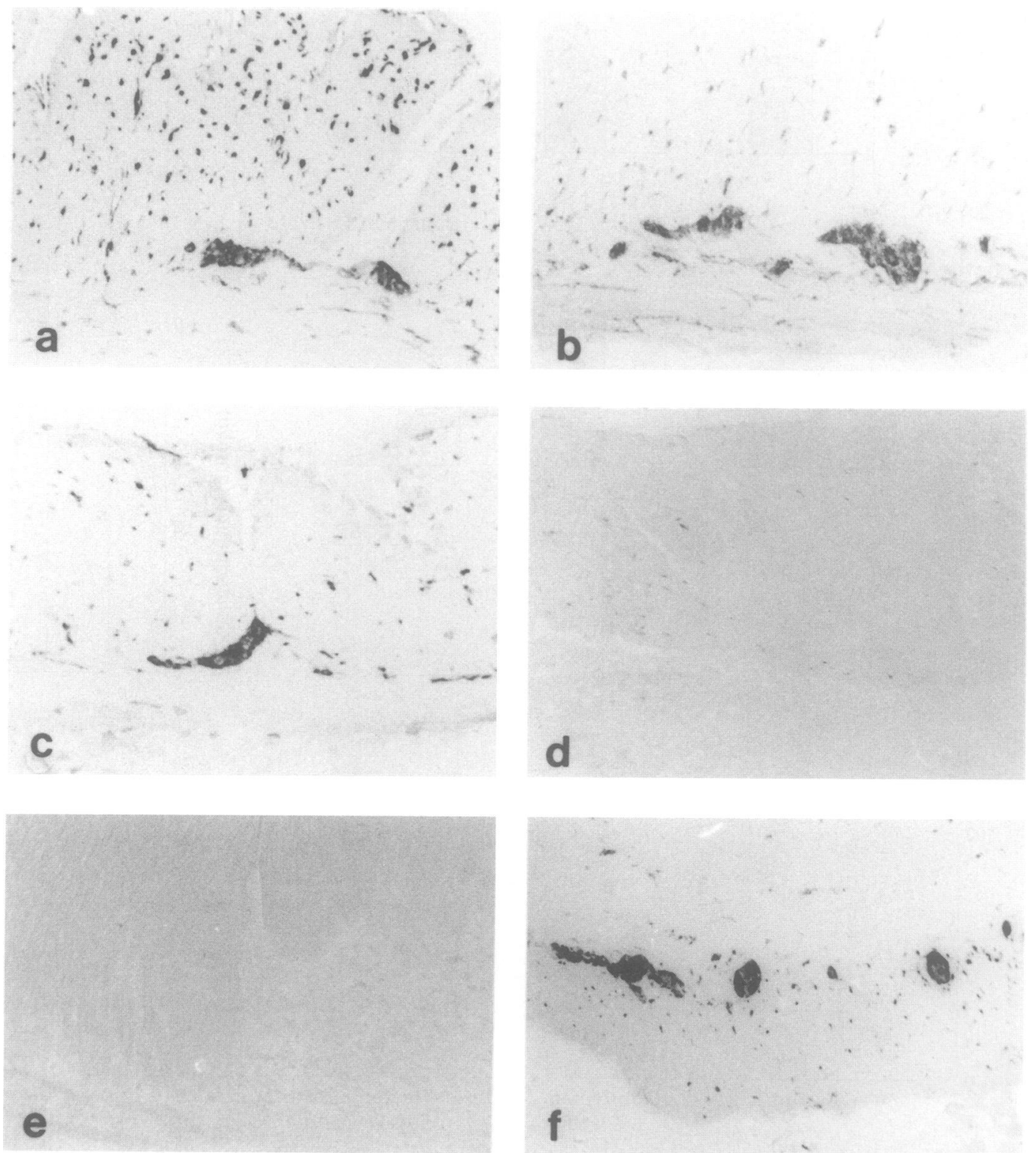

Figure 5: Representative immunoperoxidase staining for the neuronal marker PGP 9.5, showing denervation of the treated region after serosal application of $B A C$ (a,c,e, corpus; $b, d, f$, antrum). In control rats, nerve cell bodies in the myenteric plexus and nerve fibres are labelled in the corpus (a) and antrum (b). After BAC application to the antrum, neuronal labelling is present in untreated corpus (c), but is lost in the antrum (d). Similarly, after BAC application to the corpus, labelling is lost in the treated region (e) but is present in the antrum $(f)$. Original magnification, $\times 120$. 
the loss of afferent innervation. Rats that had been pretreated antrally with capsaicin did not exhibit gastric retention after fasting (fig 7). Two lines of evidence indicate that loss of the intrinsic innervation from the full radius of the antrum is associated with retention of solids. Firstly, in rats treated with BAC on one face of the antrum, with the other left intact, there was no gastric retention; secondly, application of BAC to a radial band of the corpus close to the nonglandular stomach did not produce gastric retention of solids (fig 7).

TREATMENT OF THE ANTRUM WITH BAC

DELAYS GASTRIC EMPTYING OF LIQUIDS

We studied gastric emptying of liquids in more detail in BAC treated rats in which a gastric cannula had been installed. The emptying of $0 \cdot 14 \mathrm{M}$ saline, $50 \mathrm{mM} \mathrm{HCl}$ and $4.5 \%$ peptone was significantly delayed in rats treated antrally with $0.5 \%$ BAC, compared with control rats (fig 8). It is worth noting that in control rats, emptying of $\mathrm{HCl}$ and peptone was significantly inhibited compared with saline, whereas in BAC treated rats the rates of emptying of saline, $\mathrm{HCl}$ and peptone were similar, even though all of these emptied less rapidly than in control animals. It is known that in intact rats, pre-administration of peptone just before an emptying trial with peptone (that is, peptone plus preload) produces an exaggerated inhibition of emptying owing to enhanced CCK release. ${ }^{19}{ }^{23}$ The same effect was observed in the the control rats in this study; in addition, however, peptone plus preload
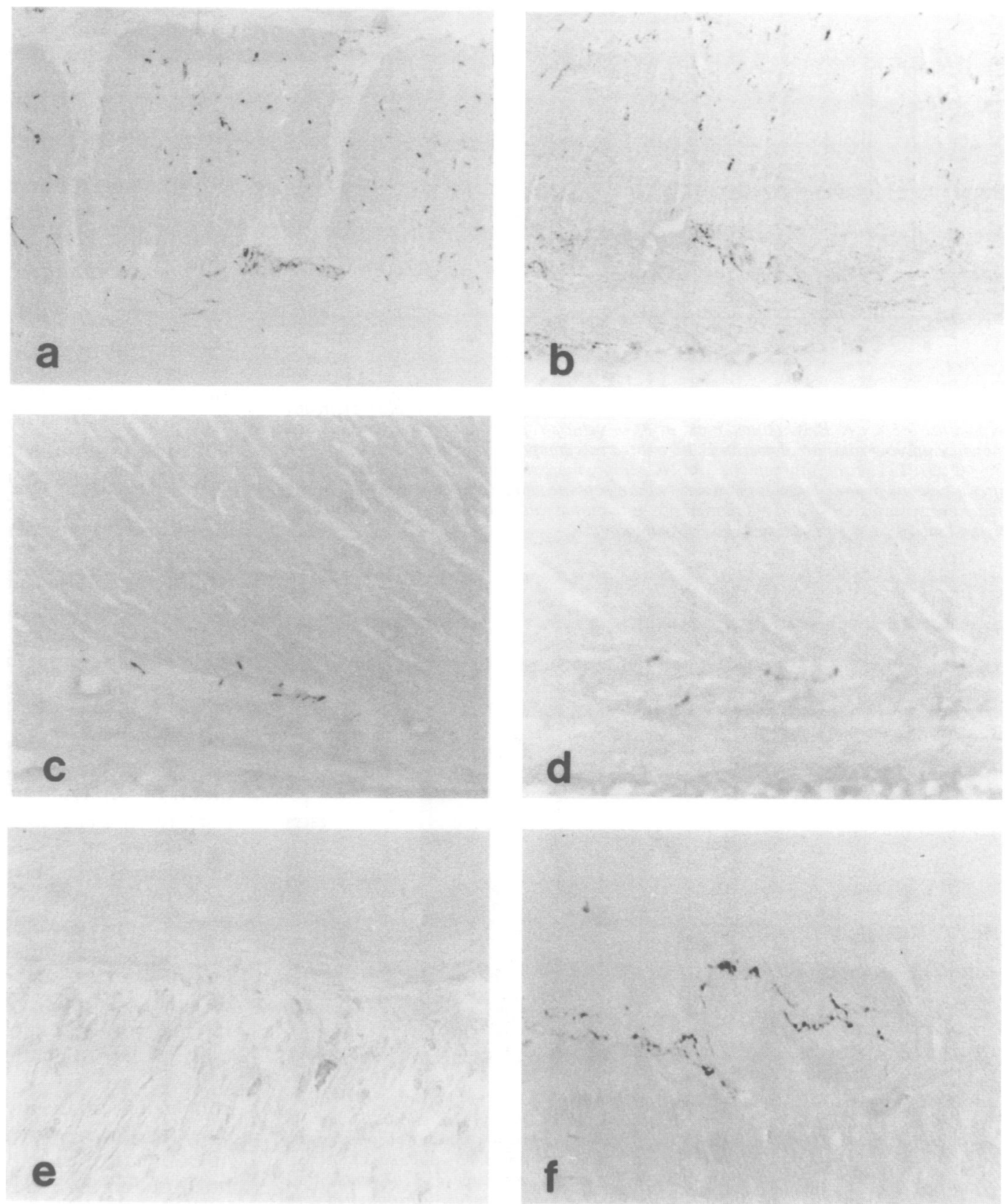

Figure 6: Representative immunoperoxidase staining for CGRP, showing loss of immunoreactivity after serosal application of $B A C$ ( $a, c, e$, corpus; $b, d, f$, antrum). In control rats, nerve fibres are labelled in the myenteric plexus and muscle layers in the corpus (a) and antrum (b). After BAC application to antrum, there is almost complete loss of immunoreactive fibres from both untreated corpus (c) and antrum (d). In contrast, after application of BAC to the corpus, immunoreactive fibres are absent in the treated region (e) but present in the antrum $(f)$. Original magnification: $a$ and $b, \times 120 ; c$ and $d, \times 190 ; e$ and $f, \times 300$. 


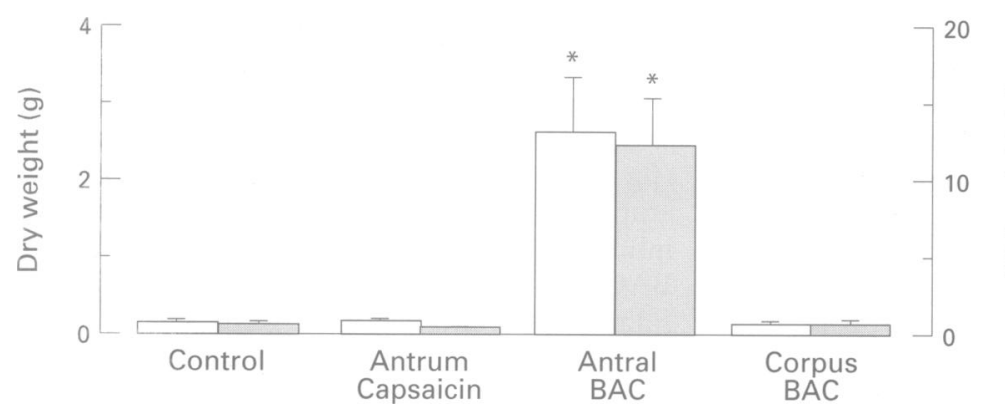

Figure 7: Gastric retention after a 48 hour fast in rats in which $B A C$ had been applied to the antrum, or to a band of corpus adjacent to the non-glandular stomach, or application of capsaicin to the antrum, or vehicle (control). Only treatment of the antrum with BAC caused gastric retention. Data shown are for wet weight (tinted bars) and dry weight (open bars) ( $n=6$ for each group; ${ }^{\star} p<0.05$, compared with vehicle, Mann-Whitney $U$ test). Values expressed as mean (SEM).

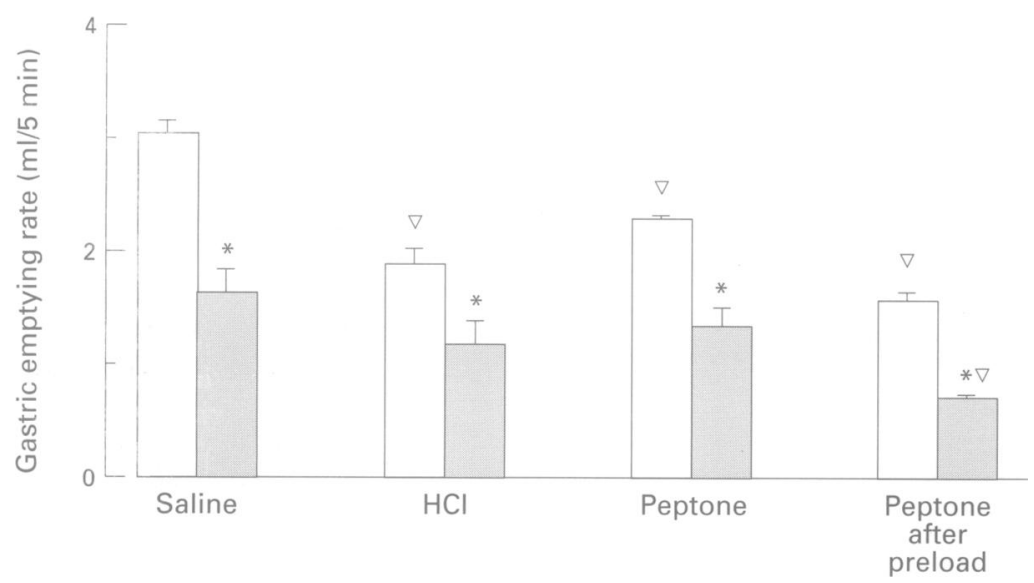

Figure 8: Gastric emptying rates for different liquids in rats with gastric fistulae pretreated with either $0.5 \%$ w/v BAC (tinted bars, $n=8$ ) or vehicle (open bars, $n=6$ ). For all liquids shown, emptying rates are slower in $B A C$ treated rats compared with control rats $\left({ }^{\star} p<0.05\right)$. In control rats, peptone and $\mathrm{HCl}$ empty more slowly than saline, and peptone after a peptone preload empties more slowly than peptone alone (inverted triangle). In BAC treated rats, saline, $\mathrm{HCl}$ and peptone empty at the same rate, but peptone after a peptone preload induces a further delay in gastric emptying. produced a significant further inhibition of emptying compared with saline in BAC treated rats (fig 8). In order to establish whether or not CCK might mediate this effect in BAC treated rats (as it does in controls), we examined gastric emptying after administration of the CCK-A antagonist L-364,718 just before the emptying trial. There was no significant difference in the emptying rate of saline after L-364,718 in BAC treated rats. Interestingly, however, the delayed emptying of peptone after a preload was significantly reversed after administration of $\mathrm{L}-364,718$ to rats treated with antral BAC, and was comparable to the emptying of saline in the same animals (fig 9).

PLASMA CCK IN BAC TREATED RATS

The lack of effect of L-364,718 on saline emptying in BAC treated rats, described earlier, suggested that the gastric retention in these animals was not primarily caused by increased release of CCK. In order to verify this conclusion we measured plasma CCK in fasted BAC treated and control rats. The mean (SEM) concentration in control rats $(1 \cdot 0(0 \cdot 1)$ $\mathrm{pM} ; \mathrm{n}=6$ ) was not significantly different from that in BAC treated rats $(1 \cdot 2(0 \cdot 1) \mathrm{pM}$; $\mathrm{n}=6$ ).

\section{Discussion}

The present study provides evidence that the integrity of the antral innervation is obligatory for the normal emptying of solids and liquids from the rat stomach. Specifically, we found that antral denervation caused by the detergent BAC resulted in retention of solids, and delayed emptying of both nutritive (peptone) and non-nutritive (saline, $\mathrm{HCl}$ ) solutions. The retention of solids and liquids could not be attributed to changes in plasma CCK, which is the hormone best known for its ability to inhibit gastric emptying, ${ }^{5}$ as concentrations of CCK in plasma were unchanged, and the CCK-A antagonist L-364,718 did not reverse the delayed emptying of saline in BAC treated rats. However, L-364,718 did reverse the inhibition of emptying of peptone after a peptone preload in BAC treated rats, suggesting that the action of CCK in controlling gastric emptying is not absolutely dependent on the antral innervation. The application of BAC to the antrum caused lesions in both the intrinsic and extrinsic afferent innervation; however, loss of the latter cannot explain the retention of solids, as there was no retention in rats treated with capsaicin which causes lesions only in extrinsic afferent fibres. Taken as a whole, therefore, the present data lead to the conclusion that the antral innervation is essential for the gastric emptying of solids and of both nutritive and non-nutritive solutions, but not for the delaying actions of CCK on gastric emptying.

The neuroendocrine mechanisms that determine gastric emptying have attracted increasing attention in recent years. ${ }^{5}$ In the case of CCK such studies have suggested a peptone in rats pretreated with $B A C$ on the serosal surface of the antrum. There was no difference in emptying rates of vehicle (hatched bars, $n=6$ ). The emptying of peptone alone was partially reversed by $L-364,718$ and the emptying of peptone after a peptone preload was significantly reversed ( $p<0.05, A N O V A)$; the latter rate was comparable with that of saline in BAC treated rats, but was still substantially slower than that of saline in control rats. 
primary site of action at receptors on vagal afferent nerve fibres, the activation of which evokes a vago-vagal reflex leading to relaxation of the gastric corpus. ${ }^{624}$ The possible involvement of the distal stomach in mediating the effect of CCK on gastric emptying has been suggested by previous studies, ${ }^{25}$ but little has been done to determine whether - in this case - CCK acts directly on smooth muscle or indirectly via the innervation. Our findings suggest that any effects of CCK on the innervation of the antro-pyloric region are not of central importance in its role as an inhibitor of gastric emptying.

The pumping action of the antrum is well known, and contributes to the episodic delivery of gastric contents to the duodenum. ${ }^{26} \mathrm{We}$ suggest that the co-ordination of this activity requires vagal efferent and intrinsic neurons and that in the absence of these neurons the antrum generates an unremitting highresistance to the passage of both liquids and solids. The effect of antral denervation on emptying can be considered specific as denervation of a fully circumferential band of proximal corpus (which would also damage vagal efferent fibres) did not lead to gastric retention. In part, the retention of solids after antral denervation may be attributable to a decreased capacity of the antrum for trituration and therefore the reduction of solids to a form suitable for emptying. In addition, however, there is also a notable delay in emptying of liquids. This effect is distinguishable from the reduced emptying of liquids seen after lesioning of afferent neurons by capsaicin ${ }^{7}{ }^{9}$ as - for example, it is only in antrally denervated rats that there is a substantial delay in the emptying of isosmotic saline. In addition to the use of BAC to study neuronal populations involved in gastric emptying, it is worth noting another, unexpected, observation made in this study. In rats treated with BAC on the serosa of the antrum there was loss of the CGRP innervation of the corpus, as well as antrum. This effect is specific in that in the converse situation (application of BAC to the serosa of the corpus) there was loss of CGRP in the treated region, but not in the antrum. The CGRP innervation of the rat stomach is entirely afferent in origin, and virtually all of it is derived from spinal afferent neurons. ${ }^{18}{ }^{27-29}$ We suggest that CGRP nerve fibres supplying the body of the stomach also supply the antrum, either by passage over the antrum or by projection of branches to both regions. Application of serosal BAC may therefore be useful in nerve-tracking studies.

A variety of experimental approaches have been used to disaggregate the mechanisms that are involved in co-ordination of gastric emptying. Analysis of flow rates across the pylorus, and simultaneous measurement of pressure waves at various recording sites in the upper gastrointestinal tract have provided valuable insight into the co-ordination of function of different regions of the stomach, pylorus and duodenum, ${ }^{2-4}$ but as yet have been less useful in elucidating neurohumoral control mechanisms. Our finding that selective lesions of defined populations of neurons in the stomach can lead to dramatic changes in gastric emptying function, suggests a useful addition to the techniques presently available for exploration of the motor control of the upper gastrointestinal tract. This type of approach may also prove useful in generating specific hypotheses as to the mechanisms of impaired emptying in clinical situations such as diabetic gastroparesis. ${ }^{30} 31$

We are grateful to the Medical Research Council for financial support. Part of this work has been presented at the AGA meeting (San Francisco 1996) and published in abstract form (Gastroenterology 1996; 110: A678).

1 Mayer EA. The physiology of gastric storage and emptying. In: Johnson LR, ed. Physiology of the gastrointestinal tract. New York: Raven Press, 1994: 929-76.

2 Houghton LA, Read NW, Heddle R, Maddern GJ, Downton J, Toouli J, et al. Motor activity of the gastric antrum, pylorus and duodenum under fasted conditions and after a liquid meal. Gastroenterology 1988; 94: 1276-84.

3 Malbert $\mathrm{CH}$, Ruckebusch Y. Relationships between pressure and flow across the gastroduodenal junction in pressure and flow across the gastroduod
$\operatorname{dogs} A m$ f Physiol 1991; 260: G653-7.

4 Prather CM, Camilleri M, Thomforde GM, Forstrom LA, Zinsmeister AR. Gastric axial forces in experimentally delayed and accelerated gastric emptying. Am $\mathcal{F}$ Physiol delayed and accelerated

5 Dockray GJ. Regulatory peptides and the neuroendocrinology of gut-brain relations. $Q \mathcal{f}$ Exp Physiol 1988; 73: 703-27.

6 Raybould HE, Tache Y. Cholecystokinin inhibits gastric motility and emptying via a capsaicin-sensitive vagal pathway in rats. Am f Physiol 1988; 255: G242-6.

7 Forster ER, Green T, Dockray GJ. Efferent pathways in the reflex control of gastric emptying in rats. Am $\mathcal{f}$ Physiol 1991; 260: G499-504.

8 Holzer HH, Turkelson CM, Solomon TE, Raybould HE. Intestinal lipid inhibits gastric emptying via CCK and a vagal capsaicin-sensitive afferent pathway in rats. $A m \mathcal{F}$ Physiol 1994; 267: G625-9.

9 Forster ER, Green T, Elliot M, Bremner A, Dockray GJ. Gastric emptying in rats: role of afferent neurons and Gastric emptying in rats: role of afferent neuron

10 Raybould HE, Roberts ME, Dockray GJ. Reflex decreases in intragastric pressure in response to cholecystokinin in rats. Am $\mathcal{F}$ Physiol 1987; 253: G165-70.

11 D'Amato M, Curro D, Montuschi P. Evidence for dual components in the non-adrenergic non-cholinergic relaxation in the rat gastric fundus: role of endogenous nitric oxide and vasoactive intestinal polypeptide. F Auto Nerv Syst 1992; 37: 175-86.

12 Desai KM, Sessa WC, Vane JR. Involvement of nitric oxide in the reflex relaxation of the stomach to accommodate food or fluid. Nature 1991; 351: 477-8.

13 Scheurer U, Varga L, Drack E, Burki HR, Halter F. Mechanism of action of cholecystokinin octapeptide on rat antrum, pylorus and duodenum. Am $¥$ Physiol 1983; 244: G266-72

14 Fox DA, Epstein ML, Bass P. Surfactants selectively ablate enteric neurons of the rat jejunum. F Pharm Exp Ther 1983; 227: 538-44

15 Dahl JL, Bloom DD, Epstein ML, Fox DA, Bass P. Effect of chemical ablation of myenteric neurons on neurotransmitter levels in the rat jejunum. Gastroenterology 1987; 92: 338-44.

16 Hutchison JB, Dimaline R, Dockray GJ. Neuropeptides in the gut: quantification and characterization of cholecystokinin octapeptide-, bombesin- and vasoactive intestinal polypeptide-like immunoreactivities in the myenteric plexus of the guinea-pig small intestine. Peptides 1981; 2: 23-30.

17 Dockray GJ, Vaillant C, Walsh JH. The neuronal origin of bombesin-like immunoreactivity in the rat gastrointestinal tract. Neuroscience 1979; 4: 1561-8.

18 Varro A, Green T, Holmes S, Dockray GJ. Calcitonin generelated peptide in visceral afferent nerve fibres: quantification by radioimmunoassay and determination of axonal transport rates. Neuroscience 1988; 26: 927-32.

19 Forster ER, Dockray GJ. The role of cholecystokinin in inhibition of gastric emptying by peptone in the rat. Exp inhibition of gastric emptying

20 Thompson RJ, Doran JF, Jackson P, Dhillon AP, Rode J. Thompson RJ, Doran JF, Jackson P, Dhillon AP, Rode J.
PGP 9.5 - a new marker for vertebrate neurons and PGP $9 \cdot 5-$ a new marker for vertebrate neuron
neuroendocrine cells. Brain Res 1983; 278: 224-8.

21 Shu S, Ju G, Fan L. The glucose oxidase-DAB-nickel method in peroxidase histochemistry of the nervous system. Neurosci Lett 1988; 85: 169-71.

22 Dimaline R, Carter N, Barnes S. Evidence for reflex adrenergic inhibition of acid secretion in the conscious rat. Am $\mathcal{F}$ Physiol 1986; 251: G615-8. 
23 Green T, Dimaline R, Peikin S, Dockray GJ. Action of the cholecystokinin antagonist L-364,718 on gastric emptying in the rat. Am f Physiol 1988; 255: G685-9.

24 Raybould HE, Gayton RJ, Dockray GJ. Mechanisms of action of peripherally administered cholecystokinin octapeptide on brain stem neurons in the rat. $\mathcal{F}$ Neurosci 1988; 8: 3018-24.

25 Yamagishi T, Debas HT. Cholecystokinin inhibits gastric emptying by acting on both proximal stomach and pylorus. Am f Physiol 1978; 234: E375-8.

26 Dent J, Sun WM, Anvari M. Modulation of pumping function of gastric body and antropyloric contractions. Dig Dis Sci 1994; 39: 28S-31S

27 Sternini C, Reeve JR, Brecha N. Distribution and characterization of calcitonin gene-related peptide immunoreactivity in the digestive system of normal and capsaicin-treated rats. Gastroenterology 1987; 93: 852-62.

28 Sternini C, Brecha N. Immunocytochemical identification of islet cells and nerve fibers containing calcitonin gene related peptide-like immunoreactivity in the rat pancreas. Gastroenterology 1986; 90: 1155-63.

29 Green T, Dockray GJ. Characterization of the peptidergic afferent innervation of the stomach in the rat, mouse and guinea-pig. Neuroscience 1988; 25: 181-93.

30 Kawagishi T, Nishizawa Y, Okuno Y, Shimada H, Inaba M, Konishi $\mathrm{T}$, et al. Antroduodenal motility and transpyloric fluid movement in patients with diabetes studied using duplex sonography. Gastroenterology 1994; 107: using

31 Horowitz M, Fraser R. Disordered gastric motor function in diabetes mellitus. Diabetologia 1994; 37: 543-51. 